\title{
Habilidades de comunicação clínica dos preceptores de medicina de família e comunidade em Florianópolis, Santa Catarina, Brasil
}

Clinical communication skills of preceptors of family and community medicine in Florianópolis, Santa Catarina, Brazil

Habilidades de comunicación clínica de los preceptores de medicina de familia y comunidad en Florianópolis, Santa Catarina, Brasil

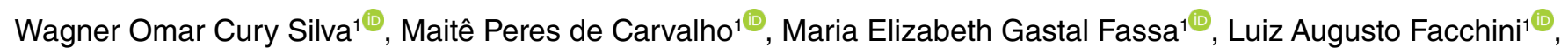
Anaclaudia Gastal Fassa ${ }^{10}$

${ }^{1}$ Universidade Federal de Pelotas, Pelotas, RS, Brasil.

\section{Resumo}

Objetivo: Este estudo investigou a autoavaliação dos preceptores (versão resumida do Guia Observacional Calgary-Cambridge) sobre quanto aplicam e ensinam HCC, bem como sobre oportunidades de ensino e qualidade do feedback. Métodos: Realizou-se um estudo transversal com 44 preceptores ativos do internato e da residência de medicina de família e comunidade de Florianópolis, Santa Catarina, Brasil. Resultados: Os preceptores se autoavaliaram positivamente em relação à aplicação e ao ensino de HCC, mas as oportunidades de ensino de HCC são escassas e a qualidade do feedbacké mediana. Conclusão: É preciso melhorar a estrutura física e a educação permanente sobre feedback e métodos de ensino, incluindo videogravação de consulta, para ampliar as oportunidades de ensino de HCC.

Palavras-chave: Educação Médica; Relações Médico-Paciente; Atenção Primária à Saúde; Comunicação em Saúde; Feedback Formativo

\begin{abstract}
Objective: This study investigated self-assessment of preceptors (short version of the CalgaryCambridge Observation Guide) about how much they apply and teach CCS, as well as about teaching opportunities and feedback quality. Methods: A cross-sectional study was carried out with 44 active preceptors of the internship and of the family and community medicine residency of Florianópolis, Santa Catarina, Brazil. Results: Preceptors self-assessed positively regarding CCS application and teaching, but CCS teaching opportunities are scarce and feedback quality is medium. Conclusion: Physical structure and permanent education on feedback and teaching methods, including video recording, need to be improved to expand CCS teaching opportunities.
\end{abstract}

Keywords: Education, Medical; Physician-Patient Relations; Primary Health Care; Health Communication; Formative Feedback

Como citar: Silva WOC, Carvalho MP, Fassa MEG, Facchini LA, Fassa AG. Habilidades de comunicação clínica dos preceptores de medicina de família e comunidade em Florianópolis, Santa Catarina, Brasil. Rev Bras Med Fam Comunidade. 2020;15(42):2673.https://doi. org/10.5712/rbmfc15(42)2673

\section{Autor correspondente:} Wagner Omar Cury Silva. E-mail: wagner.omarcs@gmail.com Fonte de financiamento: declaram não haver. Parecer CEP: não se aplica. Procedência: não encomendado. Avaliação por pares: externa.

Recebido em: 12/08/2020.

Aprovado em: 29/09/2020. 


\section{Resumen}

Objetivo: Este estudio investigó la autoevaluación de los preceptores (versión resumida del Guía Observacional Calgary-Cambridge) acerca de cuánto aplican y enseñan HCC, así como de oportunidades de enseñanza y calidad de la retroalimentación. Métodos: Se realizó un estudio transversal con 44 preceptores activos del Internado y de la Residencia de Medicina de Familia y Comunidad de Florianópolis, Santa Catarina, Brasil. Resultados: Los preceptores se autoevaluaron de forma positiva con relación a la aplicación y la enseñanza de HCC, pero las oportunidades de enseñanza de HCC son escasas, y la calidad de la retroalimentación es regular. Conclusión: Es necesario mejorar la estructura física y la educación permanente sobre retroalimentación y métodos de enseñanza, incluyendo la videograbación de consulta, para ampliar las oportunidades de enseñanza de HCC.

Palabras clave: Educación Médica; Relaciones Médico-Paciente; Atención Primaria de Salud; Comunicación en Salud; Retroalimentación Formativa

\section{INTRODUÇÃO}

As habilidades de comunicação clínica (HCC) ocupam posição central na medicina, tanto no aspecto educacional quanto assistencial, uma vez que são um elemento chave do método clínico centrado na pessoa (MCCP). Em educação médica, as HCC podem ser compreendidas como tarefas e/ou comportamentos observáveis durante a entrevista, abrangendo sete pontos principais que englobam construção da relação médico-paciente, sensibilidade para discutir com o paciente, aptidão para coletar as informações necessárias, capacidade para compreender a perspectiva do paciente, competência para compartilhar informações, maestria para acordar planos terapêuticos e perspicácia para estruturar de maneira adequada e fornecer um fechamento à consulta. ${ }^{1}$

Algumas pesquisas mostram que a excelência clínica depende de uma comunicação efetiva. ${ }^{2,3} \mathrm{O}$ uso apropriado da linguagem verbal e não-verbal favorece a criação de empatia e vínculo longitudinal, propicia um entendimento mais profundo sobre as necessidades em saúde dos usuários, facilita a construção de um plano compartilhado de cuidado e aumenta a satisfação dos usuários e profissionais, além de reduzir os erros de diagnóstico e os problemas médico-legais, garantindo melhores resultados e menores custos para o sistema de saúde. ${ }^{4-6}$

Para avaliar objetivamente as HCC, a literatura aponta alguns instrumentos, dentre os quais se destaca o Guia Observacional Calgary-Cambridge que sistematiza a avaliação de pontos importantes da consulta que podem ser generalizados para a prática clínica diária..$^{7-9}$ Esse instrumento apresenta amplo reconhecimento internacional, foi validado e está em uso em mais de uma dezena de países, de maneira que sua aplicação transpassa culturas. Além de possibilitar uma avaliação rápida, o guia pode ser utilizado em diversos contextos formativos como subsídio para nortear o feedback nas práticas de ensino e avaliação das HCC identificando lacunas na comunicação médico-paciente. ${ }^{4,5}$

A maioria dos artigos sobre HCC comparam métodos de avaliação ou avaliam habilidades específicas de comunicação, especialmente em estudantes, em contextos clínicos restritos ${ }^{8,9} \mathrm{Um}$ estudo com observação direta da consulta pelos pesquisadores indicou que as HCC de estudantes de medicina são deficientes, especialmente no que concerne à percepção da linguagem não-verbal do paciente, à estruturação e ao encerramento da consulta. ${ }^{10}$ Além disso, eles são mais estimulados a adquirir habilidades técnicas quando comparadas às habilidades emocionais, as quais reúnem competências para estabelecer contato com o paciente, demonstrando interesse por suas questões, cordialidade, empatia e assertividade. ${ }^{11}$ Estudos apontam que intervenções bem estruturadas são efetivas para melhorar as HCC. ${ }^{12}$ 
Estudos de base nacional, apontam que os preceptores, em sua maioria, não tiveram a oportunidade de aprender HCC de maneira sistemática nos seus cursos de graduação e residência. ${ }^{11}$ Um estudo holandês concluiu que a qualidade das diretrizes para comunicação médico-paciente é baixa, que elas são pouco baseadas em evidência e que não há a devida atenção à sua aplicabilidade e envolvimento do usuário. ${ }^{13}$ Outros estudos internacionais evidenciam que os médicos tendem a se autoavaliarem de maneira mais negativa do que pacientes ou pares, ${ }^{14,15}$ fato que pode sinalizar que os médicos percebem as lacunas de aprendizado de HCC em sua formação. Um estudo realizado no Reino Unido destaca a relevância da avaliação das HCC por pares, uma vez que o olhar externo amplia a perspectiva sobre os aspectos que precisam ser aprimorados. ${ }^{16}$

O sucesso da consulta clínica está diretamente relacionado à qualidade da comunicação médicopaciente. Contudo, ainda são observadas dificuldades na implementação de programas de formação que visem o desenvolvimento de competências para comunicação clínica. ${ }^{17}$ Existem controvérsias sobre como incluir as HCC nas matrizes curriculares, o que deve ser ensinado, quais métodos são mais adequados e como avaliar $^{5}$, tanto durante o processo formativo em nível de graduação, como na educação permanente dos profissionais da atenção primária à saúde (APS). ${ }^{18} \mathrm{~A}$ literatura aponta que, para promover mudanças de comportamento e atitudes dos profissionais, as abordagens educacionais das HCC devem ocorrer de maneira longitudinal e com grau crescente de complexidade, superando a aprendizagem intuitiva e por modelagem. ${ }^{18-22}$

Tendo em vista a importância das HCC nos desfechos clínicos, este estudo investiga a autoavaliação dos preceptores do internato e da residência em MFC da rede de APS de Florianópolis sobre a aplicação e o ensino das HCC, bem como sobre a qualidade do feedback. Além disso, avalia as oportunidades de ensino de HCC.

\section{MÉTODOS}

Realizou-se um estudo transversal da totalidade dos 44 preceptores ativos do internato em medicina de família e comunidade (MFC) e da residência em medicina de família e comunidade (RMFC) do município de Florianópolis/SC, no período de fevereiro a maio de 2019. Foram utilizados questionários online padronizados e pré-codificados.

A oferta da preceptoria tem condições heterogêneas quanto ao número de consultórios disponíveis, ao número de internos e residentes por preceptor e a pressão assistencial a que as equipes estão submetidas. Quando o número de consultórios disponíveis é insuficiente para a preceptoria, os internos se revezam conforme o mapa de sala (organização da utilização dos consultórios com outros profissionais), observando a consulta do preceptor ou do colega, chamando o preceptor para passar o caso ao final da entrevista. Em geral os residentes têm prioridade na utilização do consultório, sendo um dos critérios da distribuição de vagas da residência.

A residência em MFC prevê 40 horas semanais na UBS, com eventuais saídas para estágios. $O$ internato médico em MFC ocorre em imersão, com dois ciclos de 8 semanas, com 36 horas semanais cada. A pactuação dos preceptores é realizada a cada ciclo. Quanto à remuneração e vantagens, os preceptores do internato podem escolher por receber uma bolsa no valor de $\mathrm{R} \$ 550,00$ ou compensar 4 horas mensais 
para preparação de preceptoria. Os preceptores da residência recebem bolsa de $\mathrm{R} \$ 1.000,00$ reais; essas bolsas não são cumulativas. Os usuários atendidos são exclusivamente do Sistema Único de Saúde (SUS).

Os preceptores da rede docente assistencial (RDA) de Florianópolis, são médicos vinculados à prefeitura municipal de Florianópolis que trabalham na APS. Em sua maioria são egressos de residência em MFC ou médicos titulados pela Sociedade Brasileira de Medicina de Família e Comunidade (SBMFC). Apenas uma pequena parcela não tem formação específica na área.

Para a coleta dos dados foi necessária a listagem atualizada referente ao nome completo e e-mail dos preceptores da RMFC e do internato, respectivamente, as quais foram fornecidas pelo coordenador da residência da secretaria municipal de saúde do município e pelos coordenadores da disciplina do internato médico em MFC. A listagem dos preceptores do internato continha dados desatualizados, assim foi necessário buscar informações complementares com os preceptores da residência e coordenadores do internato. Em posse desses contatos foi enviando o convite para participar da pesquisa e, posteriormente, disponibilizado o link do questionário via e-mail.

O questionário incluiu, como variáveis independentes, as características demográficas (sexo e idade) e de formação dos preceptores em MFC, em saúde da família e em educação; curso de preceptoria, mestrado e doutorado; abordagem de habilidades de comunicação e realização de feedback na residência e na educação permanente; número de consultórios disponíveis para preceptoria e número de usuários atendidos pelo preceptor.

Para a caracterização do desfecho utilizou-se a versão resumida do Guia Observacional CalgaryCambridge (GOCC), a fim de captar a autoavaliação dos preceptores em relação à aplicação e ensino de HCC. Esse guia lista 28 elementos constitutivos das HCC relacionados a 6 domínios: abertura da consulta, coleta da informação e exploração de problemas, avaliação do quadro de representações do paciente, estruturação da consulta, construção da relação e encerramento da consulta.

Além disso, caracterizou-se a autoavaliação dos preceptores em relação à qualidade do feedback sobre HCC, incluindo sua especificidade em relação às HCC, caráter oportuno, solicitação de autoavaliação, capacidade de provocar reflexão sobre a prática, de indicar como melhorar e de abordar o uso de linguagem não-verbal. Descreveu-se também as oportunidades de ensino de HCC, incluindo consulta diretamente observada, observação da consulta do preceptor, análise de videogravação de consulta e indicação de bibliografia sobre HCC, nos dois meses anteriores à aplicação do questionário. Essas autoavaliações dos preceptores foram realizadas utilizando uma escala Likert de 0 a 10.

Foi realizada uma análise descritiva das variáveis independentes para caracterização da amostra e das oportunidades de ensino de HCC. Para a análise da autoavaliação dos preceptores sobre a aplicação e o ensino de HCC, e sobre a qualidade do feedback fornecido, a escala Likert foi categorizada em intervalos de 0 a 4, 5 a 7, 8 a 10 buscando caracterizar baixo, médio ou alto desempenho. Foi também calculada média, mediana e desvio-padrão para cada item da escala, bem como para o total de cada domínio e para o total da escala.

Considerando o número de sujeitos do estudo e a distribuição assimétrica dos desfechos avaliouse a associação entre os aspectos demográficos e de formação com as autoavaliações dos preceptores realizando a comparação entre as médias através do teste não-paramétrico de Wilcoxon-Mann-Whitney 
para variáveis independentes com duas categorias de resposta e de Kruskal-Wallis com teste de Bonferroni, para três ou mais categorias de resposta.

Foi obtida a carta de anuência da Universidade Federal de Santa Catarina e da secretaria municipal de saúde de Florianópolis. O projeto foi aprovado pelo Comitê de Ética em Pesquisa da Faculdade de Medicina da Universidade Federal de Pelotas, com parecer $n^{\circ} 3.086 .780$ de dezembro de 2018 . No convite e no termo de consentimento livre e esclarecido (TCLE) foi explicado aos preceptores o tema da pesquisa, tendo sido garantido o direito a não participação e também à confidencialidade das informações prestadas. O TCLE foi disponibilizado on-line e somente após o aceite foi garantido acesso ao questionário.

\section{RESULTADOS}

Foram estudados 44 preceptores, 16 eram preceptores exclusivos do internato, 10 eram exclusivos da residência e 18 atuavam em ambas, obtendo uma taxa de resposta de $92 \%$. As perdas foram concentradas nos preceptores do internato. Mais da metade dos preceptores têm entre 30 e 39 anos e 55\% são do sexo masculino. Em relação à formação, $95 \%$ tem título de especialista em MFC, sendo que destes $86 \%$ obteve o título por meio da residência. Entre os preceptores, 34\% cursou a especialização em saúde da família, $23 \%$ fez especialização na área de educação, $82 \%$ fez algum curso de preceptoria, $16 \%$ tem mestrado e $4 \%$ tem doutorado. Durante a residência, a maioria dos preceptores teve oportunidade de estudar HCC (84\%) e a forma de realizar feedback (63\%). No entanto, em educação permanente, o percentual de preceptores que estudou essas temáticas foi de $50 \%$ e $42 \%$, respectivamente. Entre os preceptores, $93 \%$ tinha mais de um consultório (não exclusivo) disponível para preceptoria e $70 \%$ atendia, em média, mais de 12 usuários por turno (Tabela 1).

A autoavaliação dos preceptores seguindo o Guia Observacional Calgary-Cambridge quanto à aplicação e ao ensino das HCC teve medianas entre 8 e 10, tanto nos itens individuais, quanto em cada domínio; e, no total, exceto pelo item relativo a "ensinar o aluno a progredir usando sentenças de transição" que teve mediana 7,5 . Os domínios com medianas iguais ou inferiores a 8,5 , tanto na dimensão aplica quanto ensina, foram "avaliação do quadro de representação do paciente", "estruturação da consulta" e "encerramento da consulta". A mediana geral da dimensão aplica foi 8,7 enquanto da dimensão ensina foi 8,4 (Tabelas 2 e 3).

$\mathrm{Na}$ aplicação das HCC, os itens que concentraram mais de $4 \%$ das respostas na categoria 0 a 4 , correspondente ao que menos realiza na escala Likert, foram: "apresentar-se para o paciente", "identificar e confirmar a lista de problemas", "negociar a agenda com o paciente", "progredir usando sentenças de transição" e "encerrar a consulta com um sumário breve" (Tabela 2). Ensinar o aluno a "progredir usando sentenças de transição", "incitar verbal e não verbalmente o paciente a responder às questões", "responder as 'deixas' verbais e não-verbais do paciente", "negociar a agenda com o paciente", "realizar comentários e questões de fácil compreensão durante as consultas", "encerrar a consulta com um sumário breve" e "avaliar as ideias do paciente quanto à causa dos problemas" concentraram mais de $6 \%$ das respostas na categoria 0 a 4 da escala Likert. Além dessas, tiveram média dos escores abaixo de 8, "ensinar o aluno a identificar e confirmar a lista de problemas" e "evitar que a leitura ou escrita interfira na comunicação com o paciente" (Tabela 3). 
Tabela 1. Aspectos demográficos, de estrutura dos serviços e de formação dos preceptores de medicina de família e comunidade do município de Florianópolis/SC, $2019(\mathrm{n}=44)$.

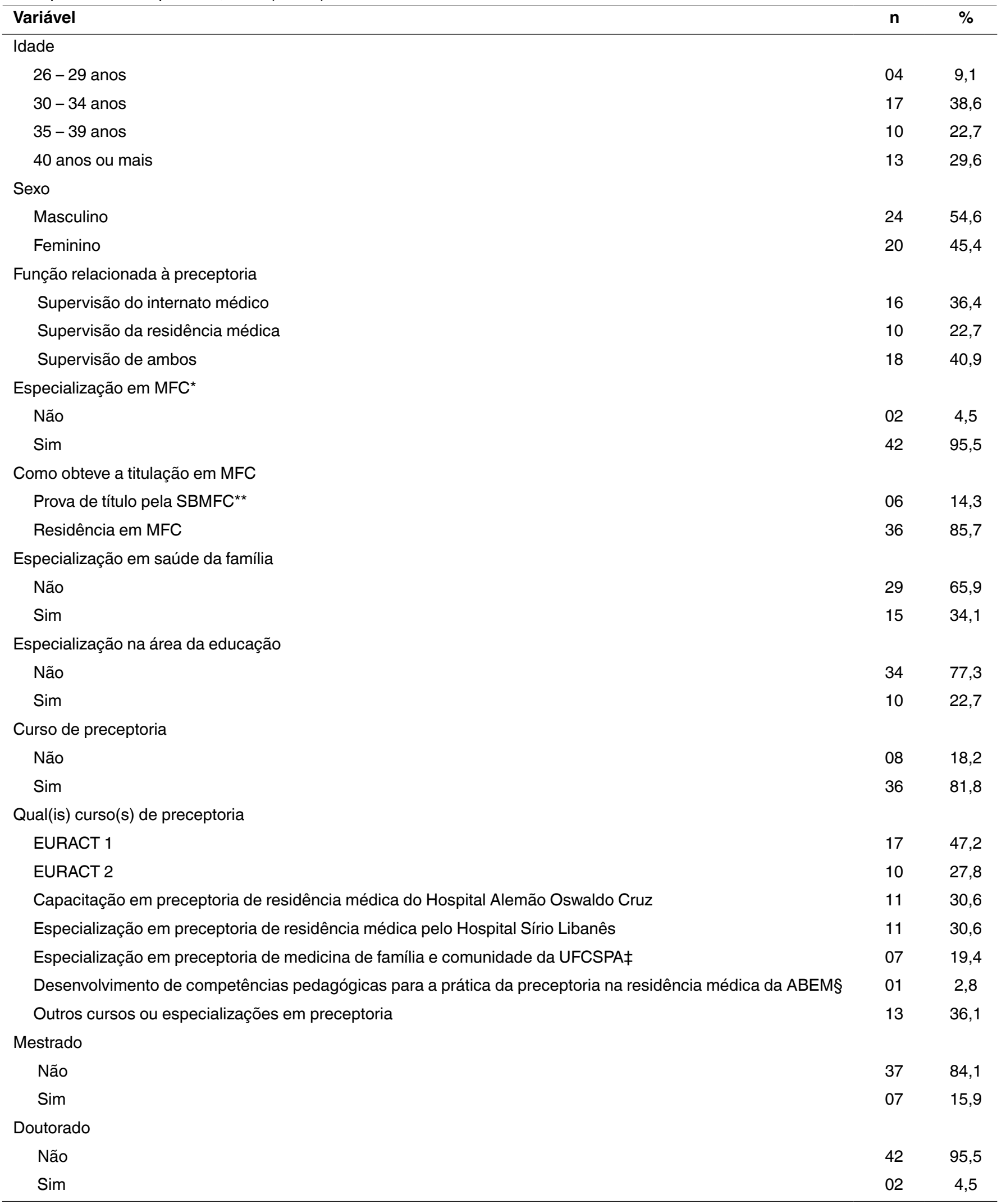




\begin{tabular}{|c|c|c|}
\hline \multicolumn{3}{|c|}{ Abordou o tema habilidades de comunicação na residência† } \\
\hline Não & 06 & 15,8 \\
\hline Sim & 32 & 84,2 \\
\hline \multicolumn{3}{|c|}{ Abordou o tema habilidades de comunicação na educação permanente† } \\
\hline Não & 19 & 50,0 \\
\hline Sim & 19 & 50,0 \\
\hline \multicolumn{3}{|c|}{ Abordou a forma de realizar feedback na residência† } \\
\hline Não & 14 & 36,8 \\
\hline Sim & 24 & 63,2 \\
\hline \multicolumn{3}{|c|}{ Abordou a forma de realizar feedback na educação permanente† } \\
\hline Não & 22 & 57,9 \\
\hline Sim & 16 & 42,1 \\
\hline \multicolumn{3}{|c|}{ Consultórios disponíveis para preceptoria } \\
\hline Um & 03 & 6,8 \\
\hline Dois & 28 & 63,6 \\
\hline Três ou mais & 13 & 29,6 \\
\hline \multicolumn{3}{|c|}{ Pacientes atendidos pelo preceptor, em média, por turno } \\
\hline $6-12$ & 13 & 29,5 \\
\hline $13-15$ & 15 & 34,1 \\
\hline $16-26$ & 16 & 36,4 \\
\hline
\end{tabular}

*MFC: Medicina de família e comunidade; **SBMFC: Sociedade Brasileira de Medicina de Família e Comunidade; *UFCSPA: Universidade Federal de Ciências da Saúde de Porto Alegre; §ABEM: Associação Brasileira de Educação Médica; ${ }^{\dagger} 6$ Perdas.

Em relação às oportunidades de ensino de HCC, nos dois meses anteriores à entrevista, metade dos preceptores utilizou consulta diretamente observada menos de 3 vezes, $24 \%$ proporcionou que o aluno observasse seu atendimento menos de 3 vezes e $92 \%$ não utilizou análise de videogravação de consulta. Dos preceptores avaliados, 97\% indica bibliografia sobre HCC, principalmente livro (81\%) e artigo (59\%) (Tabela 4).

Os itens de autoavaliação dos preceptores sobre feedback em HCC tiveram medianas entre 6,5 e 8. Os itens com maior número de preceptores na pontuação 0 a 4 na escala Likert foram a "abordagem da linguagem não-verbal” (27\%), "início do feedback solicitando uma autoavaliação do aluno" (20\%) e o "tratamento de maneira específica das HCC do aluno" (16\%). A mediana geral do feedback foi 7,3 (Tabela 5).

A Tabela 6 mostra que não há diferença significativa entre as médias das dimensões aplica e ensina do Guia Calgary-Cambridge e o feedback segundo os aspectos demográficos, de formação dos preceptores e da estrutura para preceptoria, exceto pela associação direta entre número de consultórios disponíveis para preceptoria e a qualidade do feedback.

\section{DISCUSSÃO}

O estudo aponta que os preceptores do internato e da residência de MFC de Florianópolis/SC se autoavaliam de forma bastante positiva em relação à aplicação e ao ensino de HCC, de acordo com o Guia Observacional Calgary-Cambridge. Entretanto, as oportunidades de ensino de HCC como consulta diretamente observada e a observação de consulta do preceptor são escassas e a videogravação de consulta praticamente não é utilizada. Além disso, a autoavaliação aponta uma qualidade mediana do feedback. 
Tabela 2. Autoavaliação dos preceptores de medicina de família e comunidade do município de Florianópolis/SC quanto à aplicação de habilidades de comunicação clínica segundo o Guia Observacional Calgary-Cambridge 2019 ( $n=44)$.

\begin{tabular}{|c|c|c|c|c|c|c|}
\hline \multirow{2}{*}{$\begin{array}{l}\text { Dimensão: aplica } \\
\text { domínios/itens do Calgary-Cambridge }\end{array}$} & \multicolumn{3}{|c|}{$\begin{array}{l}\text { Resposta por intervalos } \\
\text { da escala Likert (\%) }\end{array}$} & \multicolumn{3}{|c|}{$\begin{array}{l}\text { Medidas de } \\
\text { tendência central }\end{array}$} \\
\hline & $0-4$ & $5-7$ & $8-10$ & Média & Mediana & $\mathbf{D P}^{*}$ \\
\hline Domínio 1. Abertura da consulta & 2,7 & 13,2 & 84,1 & 8,9 & 9,0 & 0,86 \\
\hline Você saúda o paciente? & 0,0 & 0,0 & 100,0 & 9,7 & 10,0 & 0,67 \\
\hline Você se apresenta ao paciente? & 4,5 & 15,9 & 79,6 & 8,6 & 10,0 & 1,96 \\
\hline Você demonstra respeito pelo paciente? & 0,0 & 0,0 & 100,0 & 9,7 & 10,0 & 0,59 \\
\hline Você identifica e confirma a lista de problemas? & 4,6 & 34,1 & 61,3 & 8,0 & 8,0 & 2,00 \\
\hline $\begin{array}{l}\text { Você negocia a agenda com o paciente (acorda com o paciente os } \\
\text { temas que tratará na consulta)? }\end{array}$ & 4,6 & 15,9 & 79,5 & 8,4 & 9,0 & 1,81 \\
\hline Domínio 2. Coleta de informação - exploração de problemas & 0,3 & 10,1 & 89,6 & 8,9 & 8,9 & 0,78 \\
\hline Você incita o paciente a contar sua história? & 0,0 & 6,8 & 93,2 & 9,1 & 10,0 & 1,15 \\
\hline $\begin{array}{l}\text { Você se move adequadamente das perguntas abertas para as } \\
\text { fechadas? }\end{array}$ & 0,0 & 6,8 & 93,2 & 8,9 & 9,0 & 1,03 \\
\hline Você realiza escuta ativa dos pacientes? & 0,0 & 4,6 & 95,4 & 9,3 & 10,0 & 0,86 \\
\hline $\begin{array}{l}\text { Você incita verbal e não verbalmente o paciente a responder às } \\
\text { questões? }\end{array}$ & 0,0 & 11,4 & 88,6 & 8,8 & 9,0 & 1,02 \\
\hline $\begin{array}{l}\text { Você usa comentários e questões de fácil compreensão durante as } \\
\text { consultas? }\end{array}$ & 0,0 & 9,1 & 90,9 & 9,0 & 9,0 & 1,07 \\
\hline $\begin{array}{l}\text { Você clarifica as sentenças do paciente (confirma seu } \\
\text { entendimento sobre o que o paciente traz)? }\end{array}$ & 0,0 & 18,2 & 81,8 & 8,7 & 9,0 & 1,39 \\
\hline Você tem rigor na precisão de datas dos sinais e sintomas? & 2,3 & 13,6 & 84,1 & 8,3 & 8,0 & 1,60 \\
\hline Domínio 3. Avaliação do quadro de representação do paciente & 1,2 & 11,4 & 87,4 & 8,6 & 8,5 & 0,97 \\
\hline Você avalia as ideias do paciente quanto à causa dos problemas? & 2,3 & 9,1 & 88,6 & 8,6 & 9,0 & 1,27 \\
\hline Você avalia as preocupações do paciente frente aos problemas? & 0,0 & 9,1 & 90,9 & 8,7 & 9,0 & 1,07 \\
\hline Você incita o paciente a demonstrar suas emoções? & 0,0 & 13,6 & 86,4 & 8,7 & 9,0 & 1,19 \\
\hline Você responde às "deixas" verbais e não-verbais do paciente? & 2,3 & 13,6 & 84,1 & 8,4 & 8,0 & 1,24 \\
\hline Domínio 4. Estruturação da consulta & 4,0 & 19,3 & 76,7 & 8,2 & 8,3 & 1,10 \\
\hline $\begin{array}{l}\text { Você sumariza as informações coletadas e confirma com o } \\
\text { paciente? }\end{array}$ & 2,3 & 15,9 & 81,8 & 8,5 & 9,0 & 1,49 \\
\hline Você progride usando sentenças de transição? & 11,4 & 29,6 & 59,0 & 7,2 & 8,0 & 2,27 \\
\hline Você estrutura a consulta segundo uma sequência lógica? & 0,0 & 9,1 & 90,9 & 8,8 & 9,0 & 1,19 \\
\hline Você faz gestão adequada do tempo de consulta? & 2,3 & 22,7 & 75,0 & 8,2 & 8,0 & 1,52 \\
\hline Domínio 5. Construção da relação & 0,5 & 8,7 & 90,8 & 8,8 & 8,7 & 0,71 \\
\hline Você demonstra comportamento não-verbal adequado? & 0,0 & 13,6 & 86,4 & 8,4 & 8,0 & 1,02 \\
\hline $\begin{array}{l}\text { Você evita que a leitura ou escrita interfira na comunicação com o } \\
\text { paciente? }\end{array}$ & 2,3 & 20,5 & 77,2 & 8,2 & 8,0 & 1,49 \\
\hline Você evita fazer juízo de valor, julgar o paciente? & 0,0 & 4,6 & 95,4 & 9,2 & 9,0 & 0,93 \\
\hline Você empatiza com o paciente e o apoia? & 0,0 & 2,3 & 97,7 & 9,1 & 9,0 & 0,87 \\
\hline
\end{tabular}




\begin{tabular}{lcccccc}
\hline Você demonstra confiança? & 0,0 & 2,3 & 97,7 & 9,1 & 9,0 & 0,91 \\
Domínio 6. Encerramento da consulta & 3,0 & 16,7 & 80,3 & 8,4 & 8,5 & 1,16 \\
Você encoraja o paciente a discutir mais algum ponto adicional? & 2,3 & 9,1 & 88,6 & 8,6 & 9,0 & 1,53 \\
Você encerra a consulta com um sumário breve? & 6,8 & 27,3 & 65,9 & 7,7 & 8,0 & 2,00 \\
Você acorda com o paciente os passos seguintes? & 0,0 & 13,6 & 86,4 & 8,8 & 9,0 & 1,05 \\
Média geral dos domínios - dimensão: aplica & 2,0 & 13,2 & 84,8 & 8,6 & 8,7 & 0,72 \\
\hline
\end{tabular}

${ }^{*}$ DP: Desvio padrão.

Tabela 3. Autoavaliação dos preceptores de medicina de família e comunidade do município de Florianópolis/SC quanto ao ensino das habilidades de comunicação clínica segundo o Guia Observacional Calgary-Cambridge, 2019 ( $n=44)$.

\begin{tabular}{|c|c|c|c|c|c|c|}
\hline \multirow{2}{*}{$\begin{array}{l}\text { Dimensão: ensina } \\
\text { domínios/itens do Calgary-Cambridge }\end{array}$} & \multicolumn{3}{|c|}{$\begin{array}{l}\text { Resposta por intervalos } \\
\text { da escala Likert (\%) }\end{array}$} & \multicolumn{3}{|c|}{$\begin{array}{l}\text { Medidas de } \\
\text { tendência central }\end{array}$} \\
\hline & $0-4$ & $5-7$ & $8-10$ & Média & Mediana & $\mathrm{DP}^{*}$ \\
\hline Domínio 1. Abertura da consulta & 4,1 & 17,3 & 78,6 & 8,6 & 9,0 & 1,37 \\
\hline Você ensina o aluno a saudar o paciente? & 4,5 & 11,4 & 84,1 & 8,7 & 10,0 & 1,86 \\
\hline Você ensina o aluno a se apresentar ao paciente? & 2,3 & 11,4 & 86,3 & 9,0 & 10,0 & 1,60 \\
\hline Você ensina o aluno a demonstrar respeito pelo paciente? & 2,3 & 11,4 & 86,3 & 9,1 & 10,0 & 1,56 \\
\hline Você ensina o aluno a identificar e confirmar a lista de problemas? & 4,6 & 36,4 & 59,0 & 7,8 & 8,0 & 2,15 \\
\hline $\begin{array}{l}\text { Você ensina o aluno a negociar a agenda com o paciente (a } \\
\text { acordar com o paciente os temas que tratará na consulta)? }\end{array}$ & 6,8 & 15,9 & 77,3 & 8,1 & 9,0 & 2,27 \\
\hline Domínio 2. Coleta de informação - exploração de problemas & 4,2 & 17,5 & 78,3 & 8,3 & 8,6 & 1,36 \\
\hline Você ensina o aluno a incitar o paciente a contar sua história? & 2,3 & 13,6 & 84,1 & 8,7 & 9,0 & 1,71 \\
\hline $\begin{array}{l}\text { Você ensina o aluno a mover-se adequadamente das perguntas } \\
\text { abertas para as fechadas? }\end{array}$ & 2,3 & 18,2 & 79,5 & 8,3 & 8,0 & 1,70 \\
\hline Você ensina o aluno a realizar escuta ativa dos pacientes? & 2,3 & 15,9 & 81,8 & 8,8 & 9,0 & 1,59 \\
\hline $\begin{array}{l}\text { Você ensina seu aluno a incitar verbal e não verbalmente o } \\
\text { paciente a responder às questões? }\end{array}$ & 9,1 & 27,3 & 63,6 & 7,7 & 8,0 & 1,95 \\
\hline $\begin{array}{l}\text { Você ensina seu aluno a realizar comentários e questões de fácil } \\
\text { compreensão durante as consultas? }\end{array}$ & 6,8 & 13,6 & 79,6 & 8,3 & 8,5 & 2,01 \\
\hline $\begin{array}{l}\text { Você ensina seu aluno a clarificar as sentenças do paciente (a } \\
\text { confirmar o entendimento dele sobre o que o paciente traz)? }\end{array}$ & 4,6 & 15,9 & 79,5 & 8,1 & 8,0 & 1,71 \\
\hline $\begin{array}{l}\text { Você ensina seu aluno a ter rigor na precisão de datas dos sinais e } \\
\text { sintomas? }\end{array}$ & 2,3 & 18,2 & 79,5 & 8,2 & 8,0 & 1,57 \\
\hline Domínio 3. Avaliação do quadro de representação do paciente & 5,7 & 18,2 & 76,1 & 8,1 & 8,4 & 1,47 \\
\hline $\begin{array}{l}\text { Você ensina seu aluno a avaliar as ideias do paciente quanto à } \\
\text { causa dos problemas? }\end{array}$ & 6,8 & 11,4 & 81,8 & 8,3 & 9,0 & 1,57 \\
\hline $\begin{array}{l}\text { Você ensina seu aluno a avaliar as preocupações do paciente } \\
\text { frente aos problemas? }\end{array}$ & 2,3 & 15,9 & 81,8 & 8,4 & 8,5 & 1,37 \\
\hline $\begin{array}{l}\text { Você ensina seu aluno a incitar o paciente a demonstrar suas } \\
\text { emoções? }\end{array}$ & 4,6 & 22,7 & 72,7 & 8,2 & 8,0 & 1,75 \\
\hline $\begin{array}{l}\text { Você ensina seu aluno a responder as "deixas" verbais e não- } \\
\text { verbais do paciente? }\end{array}$ & 9,1 & 22,7 & 68,2 & 7,5 & 8,0 & 2,25 \\
\hline
\end{tabular}




\begin{tabular}{|c|c|c|c|c|c|c|}
\hline Domínio 4. Estruturação da consulta & 5,7 & 26,2 & 68,1 & 7,9 & 8,0 & 1,35 \\
\hline Você ensina seu aluno a progredir usando sentenças de transição? & 15,9 & 34,1 & 50,0 & 6,6 & 7,5 & 2,44 \\
\hline $\begin{array}{l}\text { Você ensina seu aluno a estruturar a consulta segundo uma } \\
\text { sequência lógica? }\end{array}$ & 0,0 & 20,5 & 79,5 & 8,7 & 9,0 & 1,32 \\
\hline Domínio 5. Construção da relação & 1,8 & 20,5 & 77,7 & 8,5 & 8,6 & 1,10 \\
\hline $\begin{array}{l}\text { Você ensina seu aluno a demonstrar comportamento não-verbal } \\
\text { adequado? }\end{array}$ & 4,6 & 27,3 & 68,1 & 8,0 & 8,0 & 1,74 \\
\hline Você ensina seu aluno a empatizar com o paciente e o apoiar? & 0,0 & 9,1 & 90,9 & 9,0 & 9,0 & 1,07 \\
\hline Você ensina seu aluno a demonstrar confiança? & 0,0 & 22,7 & 77,3 & 8,5 & 9,0 & 1,50 \\
\hline Domínio 6. Encerramento da consulta & 3,0 & 25,8 & 71,2 & 8,1 & 8,2 & 1,32 \\
\hline $\begin{array}{l}\text { Você ensina seu aluno a encorajar o paciente a discutir mais algum } \\
\text { ponto adicional? }\end{array}$ & 0,0 & 27,3 & 72,7 & 8,3 & 8,5 & 1,54 \\
\hline $\begin{array}{l}\text { Você ensina seu aluno a encerrar a consulta com um sumário } \\
\text { breve? }\end{array}$ & 6,8 & 27,3 & 65,9 & 7,6 & 8,0 & 2,02 \\
\hline $\begin{array}{l}\text { Você ensina seu aluno a acordar com o paciente os passos } \\
\text { seguintes? }\end{array}$ & 2,3 & 22,7 & 75,0 & 8,3 & 8,5 & 1,49 \\
\hline
\end{tabular}

Tabela 4. Oportunidades de ensino das habilidades de comunicação dos preceptores de medicina de família e comunidade do município de Florianópolis/SC, $2019(\mathrm{n}=44)^{*}$.

\begin{tabular}{|c|c|c|}
\hline Variável & $\mathrm{n}$ & $\%$ \\
\hline Não & 05 & 13,2 \\
\hline Sim, mas nenhuma vez nos últimos dois meses & 05 & 13,2 \\
\hline 1 a 2 vezes nos últimos dois meses & 10 & 26,3 \\
\hline 10 vezes ou mais nos últimos dois meses & 06 & 15,8 \\
\hline \multicolumn{3}{|l|}{ Proporciona que o aluno observe seu atendimento } \\
\hline Não & 01 & 2,6 \\
\hline Sim, mas nenhuma vez nos últimos dois meses & 02 & 5,3 \\
\hline 10 vezes ou mais nos últimos dois meses & 12 & 31,6 \\
\hline
\end{tabular}


Utiliza análise de videogravação de consulta do residente ou interno

Não

Sim, mas nenhuma vez nos últimos dois meses

1 vez nos últimos dois meses

2 vezes nos últimos dois meses

Indica bibliografia sobre habilidades de comunicação para seus alunos

Não

Sim

Qual(is) bibliografia(s) foram indicadas nos últimos dois meses?

Livro

*6 perdas.

Entre preceptores dos programas de residência em MFC do Estado de São Paulo, $70 \%$ dos entrevistados são especialistas em MFC, sendo que $57 \%$ obteve a titulação através da residência. ${ }^{23} \mathrm{~A}$ política de provimento de profissionais médicos para a rede de APS em Florianópolis requer o título de especialista em MFC, parte significativa dos egressos do programa de residência municipal acabam ficando na rede e compondo o quadro de preceptores, resultando em um maior número de profissionais com esta qualificação do que em outros municípios brasileiros. Além disso, houve estímulo aos médicos da rede para que frequentassem cursos com enfoque na formação de preceptores, que abordaram as $\mathrm{HCC}$ e a forma de realizar feedback, contrastando com a literatura que aponta falta de oportunidade formativa sobre HCC para os preceptores, ${ }^{24}$ mas em sintonia com uma tendência nacional. ${ }^{23}$

Os achados da autoavaliação nos diferentes domínios são consistentes com os de Ochoa, em $2019,{ }^{10}$ que realizou pesquisa com internos do último ano do curso de medicina, de três universidades que estagiam no Hospital Vicente Corral Moscoso. O estudo de Taylor et al., em 2018, ${ }^{25}$ na Austrália, apontaram a avaliação deficitária na estruturação da consulta, particularmente com relação à progressão utilizando frases de transição. No presente estudo, observou-se que o pior desempenho na estruturação da consulta e na compreensão da perspectiva do paciente preocupam pela centralidade desses domínios no MCCP, que abarcam aspectos longitudinais, permeando todas as etapas da consulta e realizando a ligação entre elas.

Consistente com a literatura, este estudo aponta a negociação de agenda como um ponto crítico do domínio abertura de consulta. Em estudo realizado na Alemanha com estudantes de medicina do terceiro ano, além deste item ter apresentado menor pontuação, foi também o que menos sofreu mudança após intervenção educacional de 12 semanas. ${ }^{12}$ Outro estudo realizado no Equador, com alunos do internato médico, concluiu que $74 \%$ dos internos avaliados não negociavam agenda. ${ }^{10}$ Outro item com pontuação insatisfatória no estudo da Alemanha foi avaliar as ideias do paciente quanto à causa dos problemas, que no presente estudo também esteve entre os piores desempenhos na dimensão ensina. ${ }^{12}$ 
Tabela 5. Autoavaliação dos preceptores de medicina de família e comunidade do município de Florianópolis/SC quanto ao feedback em habilidades de comunicação clínica, 2019 ( $n=44)$.

\begin{tabular}{|c|c|c|c|c|c|c|}
\hline \multirow{2}{*}{$\begin{array}{l}\text { Autoavaliação sobre feedback em } \\
\text { habilidades de comunicação clínica }\end{array}$} & \multicolumn{3}{|c|}{$\begin{array}{l}\text { Resposta por intervalos } \\
\text { da escala Likert (\%) }\end{array}$} & \multicolumn{3}{|c|}{$\begin{array}{l}\text { Medidas de } \\
\text { tendência central }\end{array}$} \\
\hline & $0-4$ & $5-7$ & $8-10$ & Média & Mediana & $\mathrm{DP}^{*}$ \\
\hline $\begin{array}{l}\text { Feedbacks são realizados imediatamente após a } \\
\text { consulta ter sido realizada? }\end{array}$ & 13,6 & 54,6 & 31,8 & 6,5 & 7,0 & 1,87 \\
\hline $\begin{array}{l}\text { Feedbacks tratam de maneira específica das } \\
\text { habilidades de comunicação do aluno no contexto } \\
\text { de uma consulta? }\end{array}$ & 15,9 & 29,6 & 54,5 & 6,9 & 8,0 & 2,36 \\
\hline $\begin{array}{l}\text { Feedbacks iniciam solicitando uma autoavaliação } \\
\text { do aluno sobre dificuldades encontradas na } \\
\text { comunicação clínica? }\end{array}$ & 20,5 & 29,5 & 50,0 & 6,9 & 7,5 & 2,63 \\
\hline $\begin{array}{l}\text { Feedbacks estimulam a reflexão sobre a prática } \\
\text { de habilidades de comunicação do aluno com o } \\
\text { paciente? }\end{array}$ & 9,1 & 47,7 & 43,2 & 6,8 & 7,0 & 1,82 \\
\hline $\begin{array}{l}\text { Feedbacks apontam claramente as habilidades de } \\
\text { comunicação e os comportamentos que podem ser } \\
\text { melhorados e sugerem como fazê-lo? }\end{array}$ & 11,4 & 36,4 & 52,2 & 7,0 & 8,0 & 2,26 \\
\hline $\begin{array}{l}\text { Feedbacks abordam a linguagem não verbal, } \\
\text { como as qualidades de emissão vocal, a distância } \\
\text { espacial e os gestos e movimentos corporais? }\end{array}$ & 27,3 & 34,1 & 38,6 & 6,1 & 6,5 & 2,47 \\
\hline Média Geral & 16,3 & 38,7 & 45,0 & 6,7 & 7,3 & 1,68 \\
\hline
\end{tabular}

${ }^{\star}$ DP: Desvio padrão.

O entendimento das razões da consulta e a definição dos seus objetivos requerem a avaliação das ideias do paciente e a negociação da agenda. Elas constituem a comunicação efetiva, permitem o planejamento da consulta e o estabelecimento dos resultados que se pretende alcançar. ${ }^{26} \mathrm{~A}$ literatura aponta que a primeira queixa do paciente em geral não é a mais importante e que, em $50 \%$ das consultas de APS, o médico e o paciente não concordavam com a natureza do principal problema apresentado. ${ }^{27}$ A negociação da agenda ocorre no final da abertura da consulta e, quando a construção de uma agenda comum entre médico e paciente não é bem-sucedida, persistem incertezas do paciente com relação à adequada identificação de sua demanda, podendo bloquear a comunicação e comprometer todas as etapas subsequentes. ${ }^{11,28}$

Ensinar HCC é tarefa complexa e exige o uso de diversas metodologias educacionais como a consulta diretamente observada, de forma a proporcionar a observação de comportamento e atitudes. ${ }^{29,30} \mathrm{~A}$ limitação na oportunidade de ensino de HCC encontrada neste estudo pode ter ocorrido devido à grande demanda assistencial, bem como, limitações no espaço físico e recursos humanos.

Embora a análise de videogravação de consulta seja considerada padrão ouro na literatura ${ }^{5}$ ela praticamente não foi utilizada nos serviços. Na residência, o ensino específico das HCC inicia com quatro encontros abordando conteúdo, seguidos de 10 encontros que focalizam a análise de videogravação de consulta com metodologia de problem based interview (PBI). Este planejamento se repete no segundo ano da residência. Isto pode justificar a reduzida frequência da utilização desta ferramenta no campo de 


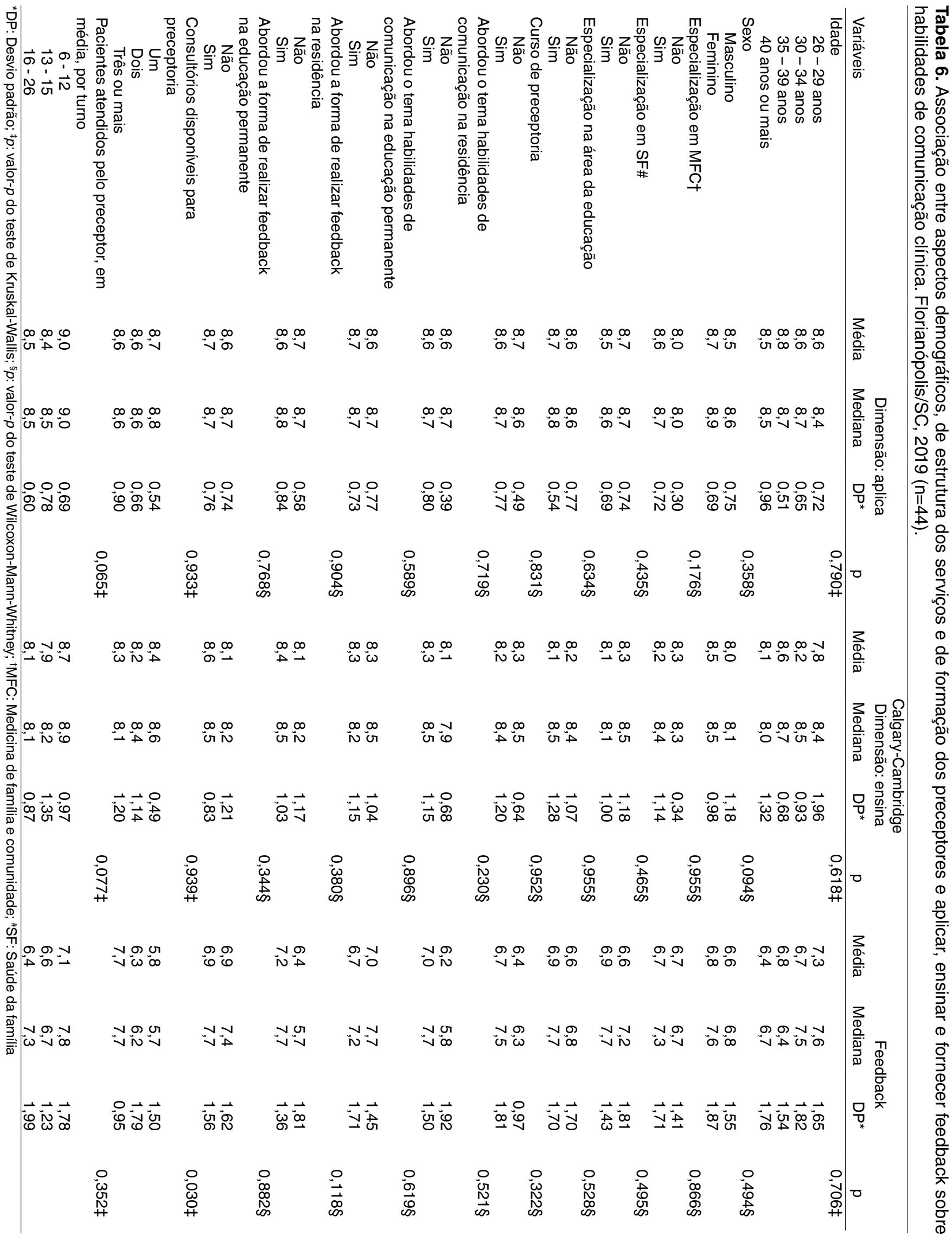


prática. No internato de MFC, os alunos têm uma aula expositiva sobre HCC com momentos de role play dos professores, possibilitando que os estudantes observem as técnicas de HCC.

O feedback é uma estratégia educacional essencial na avaliação formativa. ${ }^{31}$ Diversos autores apontam que o feedback deve iniciar por uma autoavaliação, ser respeitoso, iniciar por aspectos positivos, usar linguagem descritiva, ser específico, indicar comportamentos que podem ser melhorados, limitar o número de sugestões, ser realizado em ambiente acolhedor e próximo ao evento (oportuno) e encerrar com resumo da aprendizagem. ${ }^{11,28,31,32}$ Uma revisão sistemática evidencia que o feedback é mais efetivo quando provido por preceptores em relação longitudinal, onde se estabelece clima de confiança que facilita o diálogo baseado nas características e necessidades específicas do aluno, tornando o feedback personalizado e conectado com o plano de desenvolvimento de competências para aquele conteúdo. ${ }^{33}$

A fragilidade no feedback sobre linguagem não-verbal restringe o desenvolvimento das HCC uma vez que sinais não-verbais podem contradizer a comunicação verbal, prevalecendo a mensagem nãoverbal. ${ }^{34}$ Segundo a SBMFC, a utilização de linguagem não-verbal é a primeira da lista de competências básicas para aquisição de HCC, ${ }^{35}$ é parte central do domínio de construção da relação do GOCC e está relacionada ao estabelecimento de empatia. ${ }^{28}$ Além disso, favorece a expressão dos sentimentos, elemento fundamental do MCCP. A associação entre o número de consultórios disponíveis e o score do feedback demonstra de maneira direta a relação entre espaço físico e a qualidade do ensino em HCC, apontando a necessidade de ampliar a estrutura física das unidades docentes-assistenciais, que em geral não prevê educação em serviço.

A autoavaliação proporciona a avaliação formadora, a capacidade de o estudante fazer a metacognição, identificando os aspectos que conseguiu aprender e quais ainda precisa desenvolver, estabelecendo metas para alcançar a expertise necessária. As dificuldades encontradas neste estudo para iniciar o feedback promovendo a autoavaliação e o limitado estímulo para reflexão sobre a prática de HCC através do feedback indicam que este pode ter um caráter mais prescritivo, sendo consistente com a literatura ${ }^{21}$ que aponta falta de ensino estruturado sobre o tema. Tanto a metodologia do feedback ALOBA (agenda-led outcome-based analysis), ${ }^{7}$ usada para feedback em pequenos grupos, quanto as sessões de PBI, quando bem conduzidas, promovem a autoavaliação, uma vez que no início da atividade os estudantes devem apresentar os aspectos sobre os quais tiveram dificuldade.

Este estudo incluiu a totalidade dos preceptores de MFC de Florianópolis, com poucas perdas, obtendo uma amostra representativa da população. A aplicação on-line permite que o questionário seja preenchido no momento mais conveniente para o entrevistado, mesmo assim, foi necessário que o pesquisador insistisse na solicitação de participação na pesquisa. O questionário autoaplicado impossibilita o esclarecimento de dúvidas na hora do preenchimento podendo gerar erro de informação, porém a alta escolaridade dos entrevistados minimiza este problema. O pequeno número de participantes confere baixo poder estatístico para avaliar a diferença entre as médias, mas permite uma boa descrição das HCC nesta população. O uso do GOCC em autoavaliação deve ser examinado à luz das evidências que apontam que os profissionais se autoavaliam pior do que pacientes e pare ${ }^{15}$ e considerando que pode haver diferenças entre a autoavaliação e a aplicação, e o ensino de HCC na prática.

Considerando a importância das HCC para o MCCP são necessários estudos que avaliem a temática de forma direta, usando o GOCC como checklist e os critérios de qualidade do feedback para avaliação 
de videogravação ou observação direta de consulta, incluindo uma amostra maior de preceptores. $\mathrm{O}$ desenvolvimento de questionários para caracterizar oportunidades de ensino de HCC e qualidade do feedback são importantes contribuições deste estudo.

Futuras pesquisas devem aprofundar os motivos pelos quais as oportunidades de ensino de HCC são limitadas de forma a subsidiar a definição de estratégias para ampliá-las. É importante também validar o instrumento sobre qualidade do feedback sobre HCC para uso em estudos epidemiológicos. É necessário manter atividades de educação permanente sobre realização de feedback, aplicação e ensino de HCC, enfocando os aspectos mais frágeis apontados pelo estudo e discutindo com os preceptores as dificuldades existentes nos serviços para ampliar as oportunidades de ensino.

\section{REFERÊNCIAS}

1. Makoul G. Essential elements of communication in medical encounters: the Kalamazoo consensus statement. Acad Med. 2001 Abr;76(4):390-3.

2. Yedidia MJ, Gillespie CC, Kachur E, Schwartz MD, Ockene J, Chepaitis AE, et al. Effect of communications training on medical student performance. JAMA. 2003 Set;290(9):1157-65. DOI: https://doi.org/10.1001/jama.290.9.1157

3. Laidlaw TS, MacLeod H, Kaufman DM, Langille DB, Sargeant J. Implementing a communication skills programme in medical school: needs, assessment and programme change. Med Educ.2002 Fev;36(2):115-24. DOI: https://doi.org/10.1046/j.1365-2923.2002.01069.x

4. Moore P, Gómez G, Kurtz S, Vargas A. La comunicación médico-paciente: ¿Cuáles son las habilidades efectivas?. Rev Méd Chile. 2010 Ago;138(8):1047-54. DOI: https://doi.org/10.4067/S0034-98872010000800016

5. Kurtz S, Draper J, Silverman J. Teaching and learning communication skills in medicine. 2ª ed. Oxford: Radcliffe Publishing; 2005.

6. Maguire P, Pitceathly C. Key communication skills and how to acquire them. BMJ.2002 Set;325(7366):697-700.DOI: https://doi.org/10.1136/ bmj.325.7366.697

7. Silverman J, Kurtz S, Draper J. The Calgary-Cambridge approach to communication skills teaching: agenda-led, outcome-based analysis of the consultation. Educ Gen Pract. 1996;7:288-99.

8. Simmenroth-Nayda A, Heinemann S, Nolte C, Fischer T, Himmel W. Psychometric properties of the Calgary Cambridge guides to assess communication skills of undergraduate medical students. Int J Med Educ [Internet]. 2014 Dez; [citado 2020 Jun 17]; 5:212-8. Disponível em: https://www.ncbi.nlm.nih.gov/pmc/articles/PMC4277482/ DOI: https://doi.org/10.5116/ijme.5454.c665

9. Moral RR, Martínez FC, Leonardo CG, Monge D, Cañas F, Castaño P. Enseñary aprender habilidades de comunicación clínica en la Facultad de Medicina. La experiencia de la Francisco de Vitoria (Madrid). Educ Méd [Internet]. 2017 Out/Dez; [citado 2020 Jun 18]; 18(4):289-97. Disponível em: https://reader.elsevier.com/reader/sd/pii/S1575181317300736?token=ACBDCA1036550BE8A8EBDA04FD41425FD8 FFC3C99E98559C061A5D3397033EF9A4B4C68ADA698B3C613D78E7624C8D92 DOI: https://doi.org/1016/j.edumed.2017.03.026

10. Ochoa PDB. Evaluación de las habilidades comunicacionales de los internos rotativos de la carrera de medicina mediante la aplicación de la Guía Calgary Cambridge: Cuenca 2018 [dissertação] [Internet]. Cuenca: Universidade de Cuenca - Faculdade Ciências Médicas; 2019. Disponível em: http://dspace.ucuenca.edu.ec/handle/123456789/32570

11. Carrió FB. Entrevista clínica: habilidades de comunicação para profissionais de saúde. Porto Alegre: Artmed; 2012.

12. Simmenroth-Nayda A, Weib C, Fisher T, Himmel W. Do communication training programs improve students' communication skills? - a follow-up study. BMC Res Notes. 2012 Set;5(1):486. DOI: https://doi.org/10.1186/1756-0500-5-486

13. Veldhuijzen W, Ram PB, Van Der Weijden T, Wassink MR, Van Der Veuten C. Much variety and little evidence: a description of guidelines for doctor-patient communication. Med Educ. 2007 Jan;41(2):138-45. DOI: https://doi.org/10.1111/j.1365-2929.2006.02667.x

14. Kenny DA, Veldjuijzen W, Van DerWeijden T, Leblanc A, Lockyer J, Légaré F, et al. Interpersonal perception in the context of doctor-patient relationships: a dyadic analysis of doctor-patient communication. Soc Sci Med. 2010 Mar;70(5):763-8. DOI: https://doi.org/10.1016/j. socscimed.2009.10.065

15. Roberts MJ, Campbell JL, Richards SH, Wright C. Self-other agreement in multisource feedback: the influence of doctor and rater group characteristics. J Contin Educ Health Prof. 2013;33(1):14-23. DOI: https://doi.org/10.1002/chp.21162

16. Burt J, Abel G, Elliott MN, Elmore N, Newbould J, Davey A, et al. The evaluation of physicians' communication skills from multiple perspectives. Ann Fam Med. 2018 Jul;16(4):330-7. DOI: https://doi.org/10.1370/afm.2241 
17. Wright K, Bylund C, Ware J, Parker P, Query JL, Baile W. Medical student attitudes towards communication skills training and knowledge of appropriate provider-patient communication: a comparison of first-year and fourth-year medical students. Med Educ. 2006;11(1):4594. DOI: https://doi.org/10.3402/meo.v11i.4594

18. Cowan D, Danoff D, Davis A, Degner L, Jerry M, Kurtz S, et al. Consensus statement from the Workshop on the Teaching and Assessment of Communication Skills in Canadian Medical Schools. Can Med Assoc J [Internet]. 1992 Out; [citado 2020 Jun 20]; 147(8):1149-52. Disponível em: https://www.ncbi.nlm.nih.gov/pmc/articles/PMC1336480/pdf/cmaj00261-0055.pdf

19. Degner LF, Jerry LM, Till JE. Communication in cancer: report on the Terry Fox Workshop. Can Med Assoc J [Internet]. 1991 Jun; [citado 2020 Jun 20]; 144(11):1417-8. Disponível em: https://www.ncbi.nlm.nih.gov/pmc/articles/PMC1335673/pdf/cmaj00240-0051.pdf

20. Uhm S, Lee GH, Jin JK, Bak YI, Jeoung YO, Kim CW. Impact of tailored feedback in assessment of communication skills for medical students. Med Educ [Internet]. 2015 Jul; [citado 2020 Jul 04]; 20(1):28453. Disponível em: https://www.ncbi.nlm.nih.gov/pmc/articles/ PMC4495621/pdf/MEO-20-28453.pdf DOI: https://doi.org/10.3402/meo.v20.28453

21. Liberali R, Novack D, Duke P, Grosseman S. Communication skills teaching in Brazilian medical schools: what lessons can be learned? Patient Educ Couns. 2018 Ago;101(8):1496-9. DOI: https://doi.org/10.1016/j.pec.2017.12.021

22. Turini B, Martins Neto D, Tavares MS, Nunes SOV, Silva VLM, Thomson Z. Comunicação no ensino médico: estruturação, experiência e desafios em novos currículos médicos. Rev Bras Educ Med [Internet]. 2008 Jun; [citado ANO Mês dia]; 32(2):264-70. Disponível em: https://www.scielo.br/pdf/rbem/v32n2/a15v32n2.pdf DOI: https://doi.org/10.1590/S0100-55022008000200015

23. Ribeiro LG. Os preceptores da residência médica em medicina de família e comunidade do estado de São Paulo: quem são? Onde estão? O que fazem? [dissertação] [Internet]. Botucatu (SP): Universidade Estadual Paulista "Júlio de Mesquita Filho"; 2019. Disponível em: https://repositorio.unesp.br/handle/11449/181625

24. Kurtz SM, Silverman JD. The Calgary-Cambridge referenced observation guides: an aid to defining the curriculum and organizing the teaching in communication training programmes. Med Educ. 1996;30(2):83-9. DOI: https://doi.org/10.1111/j.1365-2923.1996.tb00724.x

25. Taylor S, Bobba S, Roome S, Ahmadzai M, Tran D, Vickers D, et al. Simulated patient and role play methodologies for communication skills training in an undergraduate medical program: Randomized, crossover trial. Educ Health [Internet]. 2018 Ago; [citado 2020 Jul 04] 31(1):10-6. Disponível em: http://www.educationforhealth.net/article.asp?issn=1357-6283;year=2018;volume=31;issue=1;spage=10;e page=16;aulast=Taylor DOI: https://doi.org/10.4103/1357-6283.239040

26. Silverman J, Kurtz S, Draper J. Skills for communicating with patients. $3^{\text {a }}$ ed. Boca Raton: CRC Press; 2013.

27. Starfield B, Wray C, Hess K, Gross R, Birk PS, D'Lugoff BC. The influence of patient-practitioner agreement on outcome of care. Am J Public Health [Internet]. 1981 Fev; [citado 2020 Jul 04]; 71(2):127-31. Available from: https://www.ncbi.nlm.nih.gov/pmc/articles/PMC1619620/ pdf/amjph00662-0017.pdf DOI: https://doi.org/10.2105/ajph.71.2.127

28. Campos CFC, Rios IC. Qual guia de comunicação na consulta médica é o mais adequado para o ensino de habilidades comunicacionais na atenção primária à saúde brasileira?. Rev Bras Educ Med [Internet]. 2018 Set; [citado 2020 Jul 04]; 42(3):108-18. Disponível em: https://www.scielo.br/pdf/rbem/v42n3/1981-5271-rbem-42-3-0108.pdf DOI: https://doi.org/10.1590/1981-52712015v42n2rb20170138

29. Shumway JM, Harden RM. AMEE guide № 25: the assessment of learning outcomes for the competent and reflective physician. Med Teach. 2003;25(6):569-84. DOI: https://doi.org/10.1080/0142159032000151907

30. Kogan JR, Holmboe ES, Hauer KE. Tools for direct observation and assessment of clinical skills of medical trainees. JAMA. 2009 Set;302(12):1316-26. DOI: https://doi.org/10.1001/jama.2009.1365

31. Borges MC, Miranda CH, Santana RC, Bollela VR. Avaliação formativa e feedback como ferramenta de aprendizado na formação de profissionais da saúde. Medicina (Ribeirão Preto) [Internet]. 2014 Nov; [citado 2020 Jul 04]; 47(3):324-31. Disponível em: http://www. revistas.usp.br/rmrp/article/view/86685/89706

32. Zeferino AMB, Domingues RCL, Amaral E. Feedback como estratégia de aprendizado no ensino médico. Rev Bras Educ Med [Internet]. 2007 Ago; [citado 2020 Jul 09]; 31(2):176-9. Disponível em: https://www.scielo.br/pdf/rbem/v31n2/08.pdf DOI: https://doi.org/10.1590/ S0100-55022007000200009

33. Veloski J, Boex JR, Grasberger MJ, Evans A, Wolfson DB. Systematic review of the literature on assessment, feedback and physicians' clinical performance: BEME guide № 7. Med Teach. 2006;28(2):117-28. DOI: https://doi.org/10.1080/01421590600622665

34. Ramos AP, Bortagarai FM. A comunicação não-verbal na área da saúde. Rev CEFAC [Internet]. 2011 Fev; [citado 2020 Jul 09]; 14(1):16470. Disponível em: https://www.scielo.br/pdf/rcefac/v14n1/186_10.pdf DOI: https://doi.org/10.1590/S1516-18462011005000067

35. Sociedade Brasileira de Medicina de Família e Comunidade (SBMFC). Currículo baseado em competências para medicina de família e comunidade [Internet]. Rio de Janeiro (RJ): SBMFC; 2014. Disponível em: http://www.sbmfc.org.br/wp-content/uploads/media/Curriculo\%20Baseado\%20em\%20Competencias(1).pdf 